\title{
MEASUREMENT OF OPENING DISPLACEMENT AND STRESS INTENSITY FACTOR OF TWO BRANCHES OF BIFURCATED NOTCH BY MOIRÉ INTERFEROMETRY
}

\author{
Shinichi Suzuki ${ }^{1}$, Mohd Azrin Bin Yusof ${ }^{2}$, Tomotaka Miyashita ${ }^{3}$ \\ 1 Toyohashi University of Technology, Department of Mechanical Engineering, Tempaku-cho, \\ Toyohashi, Aichi, 441-8580, Japan. E-mail: shinichi@me.tut.ac.jp or suzukiss1124@gmail.com \\ 2 Toyohashi University of Technology, Department of Mechanical Engineering, Tempaku-cho, \\ Toyohashi, Aichi, 441-8580, Japan. E-mail: azrinyusof5811@gmail.com \\ 3 Yamaha Motor Engineering Co. Ltd., Iwata, Shizuoka, 438-0026, Japan. E-mail:
}

\section{Introduction}

When a brittle material breaks under external force, fast propagating cracks often appear and propagate at a speed more than two hundred $\mathrm{m} / \mathrm{s}$. When crack speed is high enough, a crack bifurcates into two cracks suddenly. Since the crack bifurcation is a characteristic feature of fast propagating cracks, many researchers have been studied it, ${ }^{[1],[2]}$ however, the mechanism of the rapid crack bifurcation is not yet fully understood.

Experimental studies on crack bifurcation are classified into two groups. One is dynamic experiments that measure the rapidly bifurcating cracks directry. ${ }^{[1],[2]}$ The dynamic experiments are indispensable to figure out rapid crack bifurcation, however, measurement methods are limited.

The other is static experiments that use bifurcated notches under static or quasi-static loading. ${ }^{[3]-[5]}$ In the static experiments, one can make bifurcated notches of various shapes and can easily apply many measurement methods. Such static experiments often provide knowledge that cannot be given by the dynamic experiments. The present study is one of the static experiments.

The present study applies Moiré interferometry to measures the opening displacement of two branch notches as well as the mother notch. From the measurement results, stress intensity factors of the two branch notches are obtained. Such study has close relation to the problem of continuity of energy release rate at dynamic crack bifurcation. ${ }^{[2]}$

\section{Experimental method}

Figure 1 shows the PMMA plate specimen used in the present study. There is a bifurcated notch that has the two branches of $12 \mathrm{~mm}$. The bifurcation angle that is the half of the angle between the two branches is 13.5degrees. Diffraction grating is passed for Moiré interferfometry. Tensile force $P$ is applied to the specimen, and the opening displacement parallel to $P$ is measured by Moiré interferometry. ${ }^{[5]}$

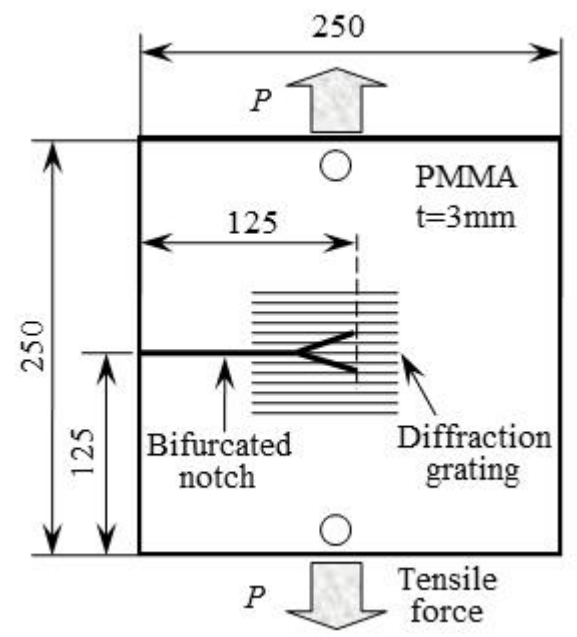

Fig. 1. PMMA plate specimen with bifurcated notch.

\section{Results}

Figure 2 shows Moiré interference fringes around the bifurcated notch. One can measure the opening displacement of the notch by counting the number of the fringes. The displacement between a fringe and the next is $1.02 \mu \mathrm{m}$.

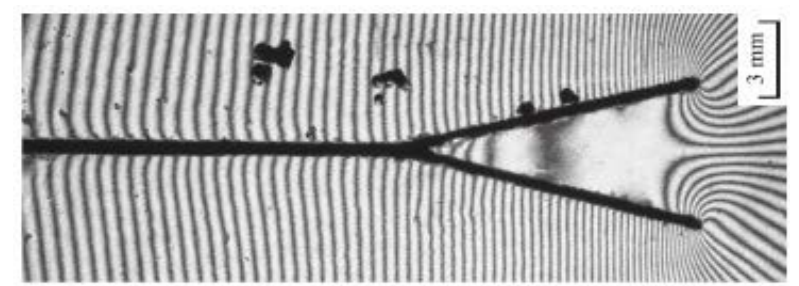

Fig. 2. Interference fringes around a bifurcated notch. 


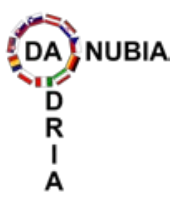

34th Danubia-Adria Symposium on Advances in Experimental Mechanics

University of Trieste, Italy, 2017
Figure 3 shows the opening displacements of the bifurcated notch measured on the photograph in Fig. 2. The opening displacement of the mother notch, $C O D_{M}$, is denoted by black circles, and is proportional to the square root of the distance $r$ from the virtual notch tip in Fig. 1.

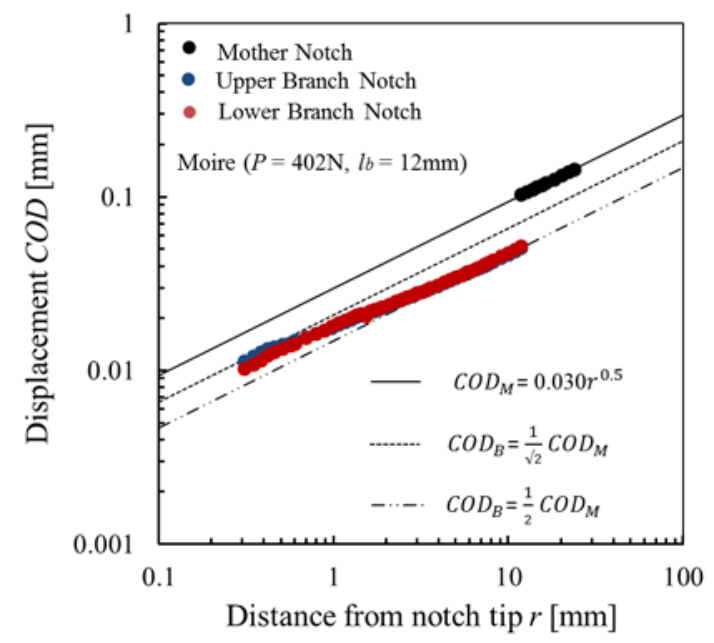

Fig. 3. Opening displacement versus $r$.

The opening displacements of the two branch notches, $C O D_{B}$, denoted by red and blue circles are a half of $C O D_{M}$ at the bifurcation point. As approaching to the tip of each branch notch, $C O D_{B}$ deviates from the line of the half of $C O D_{M}$, and approaches to the line of $C O D_{M} / \sqrt{ } 2$.

Figure 4 shows the stress intensity factors that were obtained from CODs in Fig.3 through the formula of $C O D$ in the singular stress field.

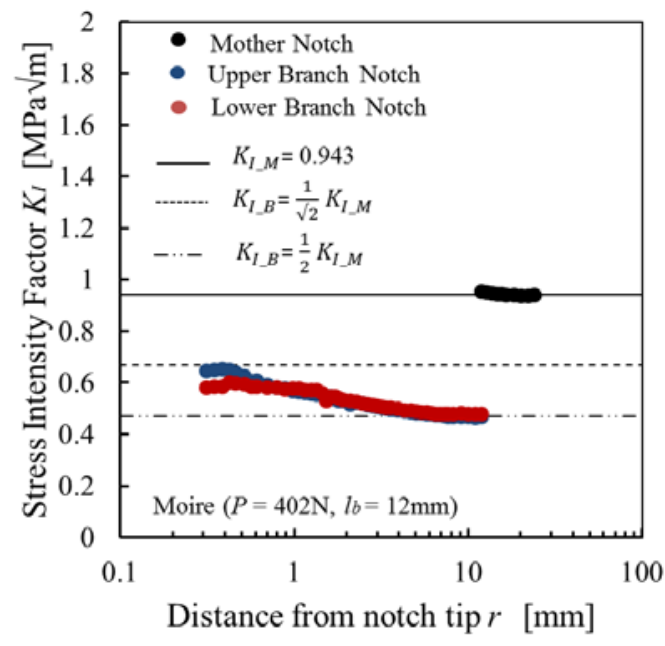

Fig. 4. Stress intensity factor versus $r$.

Stress intensity factor $K_{I M}$ of the mother crack is constant, which says the $K_{I M}$ indicates the true $K$ value of the whole bifurcated notch. ${ }^{[5]}$ Stress intensity factors $K_{I B}$ of each branch notch are the half of stress intensity factor $K_{I M}$ of the mother notch at the bifurcation point of $r=12 \mathrm{~mm}$. The $K_{I B}$ increases with getting close to the tip of the branch notch, and seems to approach the line indicating $K_{I M} / \sqrt{2}$. This is in agreement with the theoretical prediction based on energy release rate of bifurcated cracks.

\section{Remarks}

- The behavior of stress intensity factor $K_{I B}$ obtained from opening displacement $C O D_{B}$ is in agreement with the theoretical prediction based on the energy release rate of bifurcated cracks.

- Similar result is expected to appear in dynamic crack bifurcation. .

\section{Acknowledgements}

The present study was supported by Grant-inAid for Scientific Research (15K05675) by Japan Society for the Promotion of Science.

\section{References}

[1] Ravi-Chandar, K., Knauss, W. G., An Experimental Investigation into Dynamic Fracture: III. On Steady-state Crack Propagation and Crack Branching, Int. J. Fracture, 26, 1984, pp.141-154.

[2] Suzuki, S., Sakaue, K., Iwanaga, K., Measurement of Energy Release Rate and Energy Flux of Rapidly Bifurcating Crack in Homalite 100 and Araldite B by High-speed Holographic Microscopy, J. Mech. Phys. Solids, 55, 2007, pp.1487-1512.

[3] Kalthoff, J. F., On the Propagation Direction of Bifurcated Cracks. In Dynamic Crack Propagation: editor G. C. Sigh, Nordhoff, 1972, pp.449-458.

[4] Suzuki, S., Iwasaki, Y., Shimizu, Y., Yusof, M. A. B., Static Experiment on Rapid Crack Bifurcation with Y-shaped and Parallel Notches, Mech. Eng. J., 3, 2016, pp.1-12, [DOI: 10.1299/mej.16-00541].

[5] Suzuki, S., Miyashita, T., Measurement of Opening Displacement and Stress Intensity Factor of Bifurcated Notch by Moiré Interferometry, J. Solid Mech. Mater. Eng., 2, 2008, pp.25-37. 